\title{
REDUCED CORTICAL INHIBITION AND EEG RESPONSE TO TRANSCRANIAL MAGNETIC STIMULATION IN ADHD
}

Investigators at University of Heidelberg and other centers in Germany employed the N100 component of the EEG response to transcranial magnetic stimulation (TMS) as a novel marker for direct assessment of cortical inhibition in children with ADHD. Amplitude and latency of the N100 component were compared at rest, during response preparation in a motor reaction time task and during movement execution. In 20 children with ADHD compared to 19 healthy controls (8-14 years), the amplitude of N100 at rest was significantly lower and its latency tended to be shorter. During movement, N100 amplitude decreased while motor evoked potential amplitudes showed facilitation. Children with ADHD showed a smaller N100 amplitude reduction during movement execution compared with control children. The N100 amplitude evoked by TMS decreased with increasing age in both groups. The findings suggest that top-down control of motor cortical inhibition is reduced in ADHD, and the deficit in cortical control is an intrinsic qualitative deficit and not due to delayed development. (Bruckmann S, Hauk D, Roessner V, et al. Cortical inhibition in attention deficit hyperactivity disorder: new insights from the electroencephalographic response to transcranial magnetic stimulation. Brain 2012 Jul;135(Pt 7):2215-30). (Respond: Dr Stephan Bender, Child and Adolescent Psychiatric Hospital, University of Technology, Dresden, Fetscherstr. 74, D-01307 Dresden, Germany. E-mail: Stephan.bender@uniklinikum-dresden.de).

COMMENT. The authors conclude that reduced cognitive control of the motor system may contribute to diminished motor cortex inhibition and is responsible for the deficient TMS-evoked N100 amplitude reduction during motor response preparation and motor execution in ADHD. Effects of age on TMS-evoked N100 are not compatible with a delayed development of cortical inhibition but point to intrinsic inhibition deficits in the motor system of ADHD. The TMS-evoked EEG potentials are a promising new marker of cortical inhibition in children with ADHD. (Bender S et al. Electroencephalographic response to TMS in children: evidence for giant inhibitory potentials. Ann Neurol 2005 Jul;58(1):58-67). The TMS-evoked N100 is considered to be a marker of motor cortex inhibition influenced by cortico-striato-thalamo-cortical loops.

\section{MOVEMENT DISORDERS}

\section{PREVALENCE OF TIC DISORDERS: A META-ANALYSIS}

Researchers at the University of Calgary, Alberta, Canada; University of Toronto, Ontario, Canada; and Dublin, Ireland evaluated the prevalence of tic disorders by using MEDLINE and Embase databases to perform a meta-analysis of reports published 19852011. In 13 studies of children with Tourette syndrome, the prevalence was $0.77 \%$. Boys were affected more often than girls, $1.06 \%$ vs. $0.25 \%$. Transient tic disorder, the most common tic disorder, affected 3\% children. In adults, the prevalence of Tourette syndrome based on a meta-analysis of 2 studies was $0.05 \%$. The prevalence of tic disorders was higher in all studies performed in special education populations. The 
current distinction of chronic tic disorders (i.e.. Tourette syndrome, chronic motor tic disorder, and chronic vocal tic disorder) into separate categories is of debatable value. Except for the socially disabling effects of vocal tics, few differences are likely between chronic tic disorders in their etiology and response to therapy. (Knight T, Steeves T, Day L, Lowerison M, Jette N, Pringsheim T. Prevalence of tic disorders: a systematic review and meta-analysis. Pediatr Neurol 2012 Aug;47(2):77-90). (Respond: Dr Pringsheim, Dept. Clinical Neurosciences and Pediatrics, Calgary Tourette Syndrome Clinic, University of Calgary, Alberta Children's Hospital, 2888 Shaganappi Trail NW, C4-431, Calgary, Alberta T3B 6A8, Canada. E-mail: tmprings@ucalgary.ca).

COMMENT. Meta-analysis reveals that tic disorders are more common in children than in adults, in special education populations than in general populations of children, and among boys more than among girls. Transient tic disorders are the most common type of tic disorder in children, followed by chronic tic disorders and Tourette syndrome. The higher prevalence of tic disorders in boys than in girls (4:1) is a similar gender difference to that in other developmental disorders, such as autism (4 boys to 1 girl) and ADHD that affects boys 3-6 times more often than girls.

\section{ANTIEPILEPTIC DRUGS}

\section{EFFECTS OF TOPIRAMATE ON COGNITIVE FUNCTION}

Investigators at the Universities of Minnesota and Florida determined the effect of topiramate on linguistic behavior, verbal recall and working memory using a computational linguistics system for automated language and speech analysis (SALSA). Twenty-five healthy volunteers between 18 and 50 years of age at 2 study sites received either $100 \mathrm{mg}$ oral topiramate, $2 \mathrm{mg}$ oral lorazepam (active control), and placebo or topiramate and placebo in a randomized, double-blind, crossover design. Neuropsychological tests, language/verbal tests, and SALSA were performed on speech samples recorded during the tests. Topiramate plasma levels that ranged from 0.23 to $2.81 \mathrm{mcg} / \mathrm{ml}$ were associated with impairment of 1) measures of verbal fluency elicited during a picture description task, 2) correct number of words recalled on a paragraph recall test, and 3) reaction time recorded during a working memory task. The novel system of automated speech and language analysis (SALSA) allows determination of drug plasma concentration and its impact on cognitive functioning as reflected in spoken language discourse. (Marino SE, Pakhomov SVS, Han S, et al. The effect of topiramate plasma concentration on linguistic behavior, verbal recall and working memory. Epilepsy Behav 2012 Jul;24(3):365-72). (Respond: Dr SE Marino, Center for Clinical and Cognitive Neuropharmacology, University of Minnesota, 717 Delaware Street, SE, Minneapolis, MN 55414, E-mail: marin007@umn.edu).

COMMENT. The SALSA automated language analysis in healthy volunteers is thought to establish effects of a drug on cognition, natural language and speech production in the absence of cognitive impairment associated with epilepsy and its underlying brain dysfunction. The single dose test limits ability to define chronic effects. 